\title{
Um Estudante Simulado para Atuação em Ambientes Multiagente de Aprendizagem em Redes Bayesianas
}

\author{
Michele dos Santos da Silva, Rosa Maria Vicari \\ \{mssilva,rosa\}@inf.ufrgs.br
}

Resumo: Este trabalho descreve o projeto de um estudante simulado para atuação em ambientes multiagente de aprendizagem. Nesses ambientes, estudantes humanos e simulados trabalham cooperativamente, através da formação de grupos de trabalho. Por meio de um editor colaborativo, os estudantes constroem uma rede bayesiana que modela um caso de estudo. O estudante simulado auxilia o grupo a sair de um estado de estagnação na construção da rede ou faz com que o grupo reflita sobre uma determinada variável ou relação. Ele propõe questões, insere e remove arcos e nodos da rede em construção. Desse modo, utiliza uma forma de comunicação adequada ao domínio e à interação com estudantes reais, sendo visto como um companheiro e não como um assistente.

Palavras-Chave: Estudante Simulado, Ambientes Colaborativos, Redes Bayesianas.

\section{A Simulated Student for Multiagent Learning Environments on Bayesian Networks}

\author{
Michele dos Santos da Silva, Rosa Maria Vicari \\ \{mssilva,rosa\}@inf.ufrgs.br
}

\begin{abstract}
This work describes the design of a simulated student for multiagent learning environments. Human and simulated students work cooperatively, within workgroups, in order to solve a study case. Through the use of a collaborative editor, the students build a Bayesian network, which models the study case. The simulated student helps the group when a stagnation state is reached or when the group must think more about a variable or relationship. It asks questions about the network, removes and inserts arcs and nodes to the network under construction. By doing this, it uses a mean of communication suitable for the study domain and for the interaction with real students. Thus, real students can view a simulated student as a partner and not only as an assistant.
\end{abstract}

Keywords: Simulated Student, Collaborative Environments, Bayesian Networks. 


\section{Introdução}

Os sistemas computacionais atuais podem utilizar a aprendizagem de forma a adquirir conhecimentos e habilidades sofisticadas. As técnicas de aprendizagem têm sido empregadas com sucesso tanto em máquinas simbólicas como em redes neurais artificiais. Com base nisso, é possível construir sistemas computacionais que simulem estudantes humanos. Um estudante simulado é um sistema computacional com padrões de comportamento similares àqueles apresentados por estudantes humanos.

A área de educação pode obter proveito dessa tecnologia, que apresenta novas oportunidades educacionais. Professores podem desenvolver e praticar suas habilidades em estudantes simulados. Estudantes podem trabalhar colaborativamente, formando pares com estudantes simulados ou ensinando-os, caso os estudantes possuam menos conhecimento sobre determinado tema (Dillenbourg, 1999). Os desenvolvedores de materiais educacionais podem realizar testes com estudantes simulados de forma a obter uma conclusão precisa em um estágio inicial de desenvolvimento desses materiais.

Diversas arquiteturas de estudantes simulados vêm sendo desenvolvidas com o objetivo de avaliar a autoria em jogos (Virvou; Manos, 2003), de diminuir as dificuldades em ambientes colaborativos (Vizcaino; Du Boulay, 2002), de modelar o processo de raciocínio do estudante (Matsuda et. al, 2007), entre outros. As aplicações cujo objetivo é auxiliar a aprendizagem por meio da colaboração com estudantes humanos, em sua maioria, baseiam-se na comunicação textual entre estudantes reais e estudantes simulados. Para domínios em que a comunicação textual não é uma abordagem natural, esses estudantes artificiais têm aplicação restrita.

Este trabalho apresenta um estudante simulado que participa da resolução de um caso de estudo, modelado através de uma Rede Bayesiana (RB). O estudante simulado proposto explora formas de comunicação mais naturais ao domínio do problema. Ele retira e insere nodos e arcos na RB do seu grupo. Seu objetivo é auxiliar o grupo a sair de um ponto de estagnação, através da inserção de um nodo ou arco importante para a continuidade da resolução. Ele também pode remover um elemento correto, ou inserir um elemento incorreto na rede, de forma a estimular a reflexão. Além disso, ele formula questões sobre os relacionamentos existentes entre as variáveis, permitindo que os estudantes humanos reflitam e realizem alterações na RB.

Outra contribuição deste trabalho é a construção de um companheiro de aprendizagem (Holmes, 2003) para um grupo de estudantes. Os companheiros de aprendizagem existentes (Burleson, 2004), (Chou; Chan e Lin, 2002) propõem a atuação em pares. Um "par de aprendizagem" é formado por um estudante real e um estudante simulado. Neste trabalho, o estudante simulado é um companheiro de aprendizagem para todo o grupo, devendo considerar a atuação conjunta do grupo.

\section{Ambiente Multiagente Probabilístico Inteligente de Aprendizagem (AMPLIA)}

O AMPLIA (Ambiente Multiagente Probabilístico Inteligente de Aprendizagem) é um ambiente de aprendizagem voltado para a educação médica, que suporta o desenvolvimento do raciocínio e a modelagem de hipóteses diagnósticas (Vicari et al. 2003). Ele é composto por um sistema multiagente e por redes bayesianas, que vêm sendo utilizadas para modelar domínios incertos como a medicina.

No AMPLIA, o aluno modela, através de uma rede bayesiana, o diagnóstico para um estudo de caso e seu modelo é comparado ao modelo de um especialista no domínio. 
As diferenças entre as redes são tratadas por meio de estratégias pedagógicas baseadas na interação e na negociação entre os agentes inteligentes do sistema e o aluno. $\mathrm{O}$ AMPLIA tem como usuários finais alunos do curso de medicina, cujo objetivo é construir corretamente o diagnóstico para um paciente, entendendo como as diferentes variáveis (história clínica, sintomas, dados laboratoriais) relacionam-se probabilisticamente.

\subsection{Redes Probabilísticas}

As Redes probabilísticas permitem manipular a incerteza com base em princípios matemáticos fundamentados. Sua principal característica é a habilidade para reduzir o cálculo de inferência a uma série de cálculos locais, evitando o cálculo da distribuição de probabilidade conjunta global. A sua representação gráfica explicita relações de dependência e constitui uma poderosa ferramenta na representação de conhecimentos.

Redes bayesianas são grafos acíclicos orientados em que os nodos representam variáveis de interesse e os arcos representam influências causais entre as variáveis (Pearl, 1993). A cada nodo está associada uma tabela de probabilidades a priori ou condicional, que quantifica a influência dos nodos pai.

A figura 1 ilustra uma RB que descreve a relação causal entre as estações do ano (S), a ocorrência de chuvas durante a estação (C), a utilização do irrigador durante a estação (I), a umidade do pavimento (U), e o fato de o pavimento estar escorregadio (E).

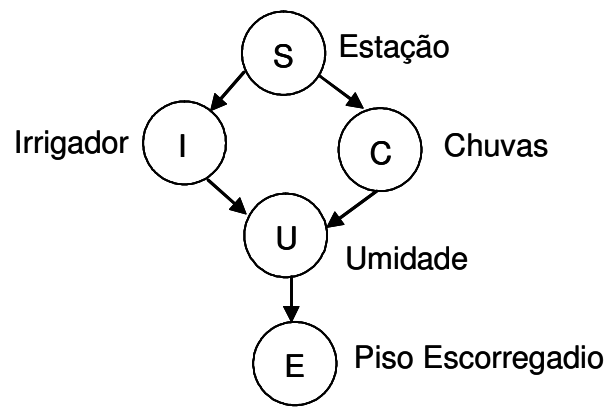

\section{Figura 1 - Uma rede bayesiana representando influências causais entre cinco variáveis.}

As redes bayesianas podem ser estendidas com nodos de utilidade e de decisão, de forma a representar os processos deliberativos de um agente autônomo. Nesse caso, são denominadas redes de decisão ou diagramas de influência.

\subsection{Sistema Multiagente AMPLIA}

O AMPLIA é composto pelos seguintes agentes artificiais: agente Aprendiz, agente de Domínio e agente Mediador (Figura 2). O agente Aprendiz representa o aluno, reunindo todas as evidências concretas sobre o estado de seu processo de aprendizagem. O agente de Domínio incorpora a base de conhecimentos sobre o tema a ser estudado. Ele é responsável pelos conteúdos e pela avaliação da qualidade da rede do aluno. $\mathrm{O}$ agente Mediador incorpora as estratégias e táticas pedagógicas necessárias ao processo de ensino-aprendizagem e decide como e quando utilizá-las. 


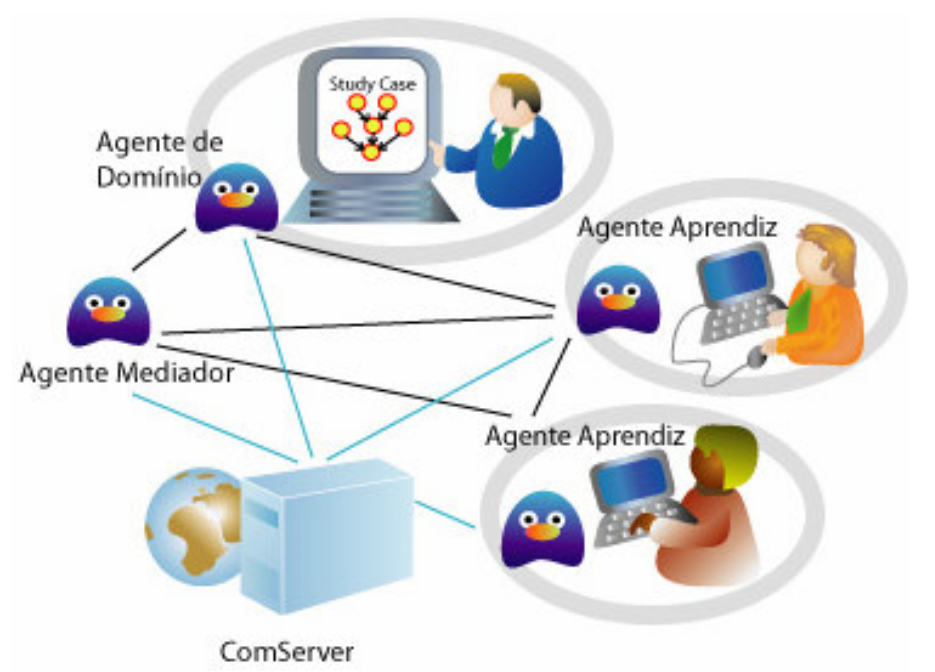

Figura 2 - Arquitetura do AMPLIA: comunicação entre o agente Aprendiz, de Domínio e Mediador.

\section{Agente de Domínio}

O Agente de Domínio tem conhecimento da área médica. Esse conhecimento é representado por RBs construídas por especialistas no domínio. Assim, o modelo do conhecimento está disponível para o estudante e pode ser explicado de forma a auxiliar o processo de aprendizagem.

O Agente de Domínio envia para o Agente Aprendiz o caso de estudo a ser modelado e a lista de variáveis que o aprendiz pode usar na construção do modelo hipotético. O Agente de Domínio recebe a rede do modelo do aprendiz e revisa as crenças do aprendiz, comparando a rede do modelo do especialista com rede do modelo do estudante. De acordo com os conflitos resultantes dessa comparação, o agente pode classificar a rede, de acordo com o apresentado na Tabela 1.

Tabela 1. Classificação da Rede Bayesiana pelo Agente de Domínio.

\begin{tabular}{|l|l|}
\hline Rede & Parâmetros \\
\hline Impossível & Rede não satisfaz a definição de uma Rede Bayesiana. \\
\hline Incorreta & Rede Bayesiana cujo modelo está conceitualmente incorreto. \\
\hline Incompleta & $\begin{array}{l}\text { A rede não apresenta alguns nós ou relações que são } \\
\text { consideradas importantes. }\end{array}$ \\
\hline Possível & $\begin{array}{l}\text { É uma rede diferente do modelo armazenado, mas satisfaz o } \\
\text { caso de estudo proposto ao aprendiz. }\end{array}$ \\
\hline Completa & É idêntica ao modelo proposto pelo especialista. \\
\hline
\end{tabular}

\section{Agente Aprendiz}

O Agente Aprendiz é responsável pela construção do modelo do estudante, por meio da observação de suas ações em um editor gráfico (Seixas et al., 2004). Essas ações podem ser observadas através de um $\log$ e são os processos de inserção e remoção de nodos e arcos durante a construção da RB. 
Através do $\log$, o Agente Aprendiz tem a possibilidade de inferir como o aluno está desenvolvendo seu modelo de RB para o problema que está sendo estudado, extrair informações específicas que ajudarão o Agente Mediador a selecionar a melhor estratégia pedagógica para uma determinada situação e identificar a necessidade de sugerir ou acionar o modo tutor.

\section{Agente Mediador}

O Agente Mediador é responsável pela mediação das interações entre o Agente Aprendiz e pelo Agente de Domínio, em cada ciclo da construção da RB.

Um diagrama de influências é responsável por realizar a tarefa de seleção das táticas que apresentarão maior relevância, em momentos diferentes da interação. Os parâmetros usados são os resultados da avaliação da RB, o nível de confiança declarado pelo estudante e a credibilidade. O Agente Mediador tem o objetivo de tornar possível a representação das dependências entre esses parâmetros e assimilar a necessidade de incorporar as mudanças constantes que ocorrem no processo de aprendizagem.

\subsection{Editor Colaborativo do AMPLIA}

De acordo com as novas propostas em ensino médico e aprendizagem baseada em problemas (Peterson, 1997), o editor do AMPLIA passou a permitir a edição colaborativa. Por meio do editor colaborativo (Figura 3), os estudantes editam a RB do grupo cooperativamente. $\mathrm{O}$ estudante solicita permissão para editar a rede e, após a edição, libera a rede para que os demais estudantes do grupo trabalhem no caso de estudo. Os estudantes se comunicam através da ferramenta de chat embutida no editor.

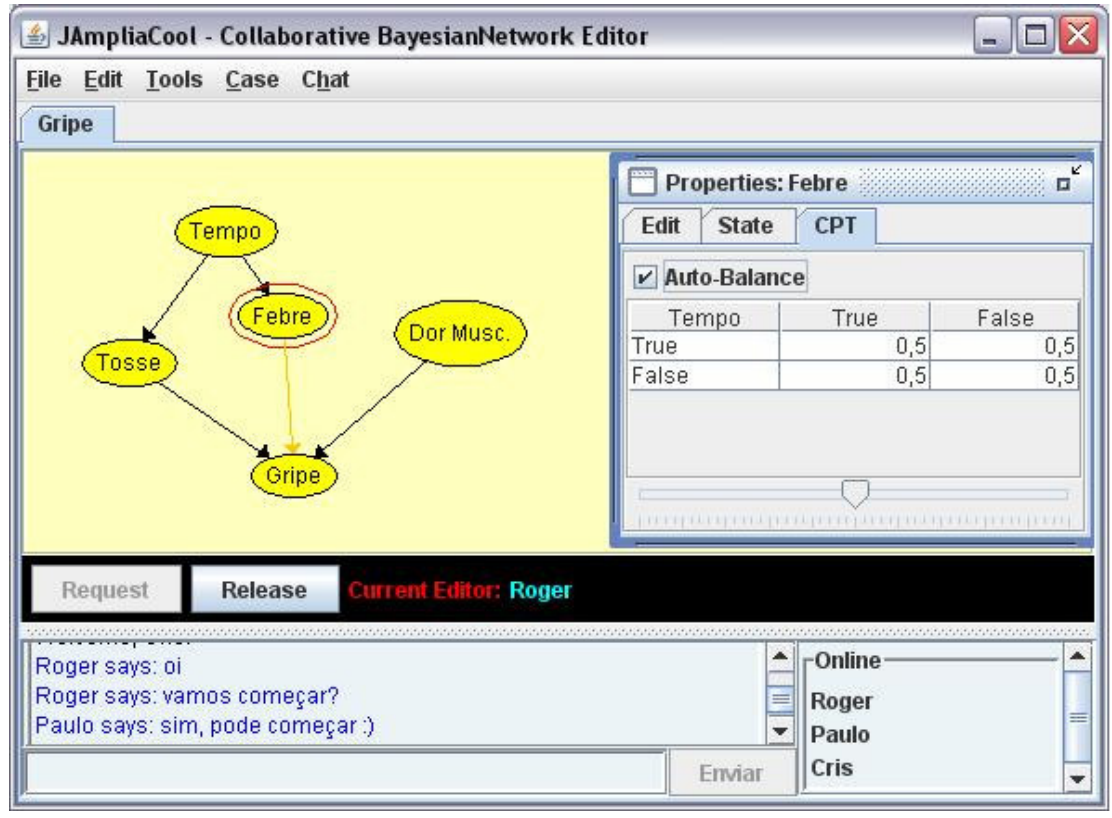

Figura 3 - 0 editor colaborativo de Redes Bayesianas.

A atividade do grupo é monitorada por um Agente Social. O objetivo principal do Agente Social (Boff, 2007) é estimular a interação dos estudantes. O Agente Social sugere grupos de trabalho para resolver tarefas cooperativamente. As ações dos estudantes são armazenadas no modelo do estudante e essa base de dados é consultada para a formação do grupo, no momento de sugerir uma interação. Os grupos são 
formados dinamicamente, de acordo com a tarefa que está sendo desenvolvida. Cada estudante pode participar de diversos grupos, de acordo com seu interesse.

\section{Integrando um Estudante Simulado no Ambiente AMPLIA}

De acordo com VanLehn e Ohlsson (1994), um estudante simulado é um agente que, para modelar um episódio de aprendizagem, recebe como entradas:

a) Uma representação formal das partes relevantes do conhecimento do estudante exatamente antes do episódio de aprendizagem;

b) Uma representação formal da instrução dada ao estudante.

Esse agente produz duas saídas:

a) Uma representação formal do conhecimento do estudante exatamente após o episódio de aprendizagem;

b) Uma representação o comportamento do estudante durante o episódio.

Para representar o conhecimento do estudante $\mathrm{e}$ as modificações no conhecimento geradas a partir das experiências de aprendizagem, são utilizadas redes bayesianas dinâmicas, que são redes bayesianas que incluem informações temporais.

Para permitir sua integração ao AMPLIA, o estudante simulado está sendo construído como um agente de software autônomo. Os agentes são sistemas que possuem a capacidade de decidir suas ações de forma a satisfazer os seus objetivos. Eles devem operar de forma robusta em ambientes abertos e imprevisíveis, em que existe uma probabilidade significativa de falha em suas ações (Wooldridge, 1999). Tabela 2.

As responsabilidades atribuídas ao Agente Estudante Simulado são descritas na

Tabela 2. Responsabilidades do Agente Estudante Simulado no contexto do Sistema Multiagente do AMPLIA.

1. Permitir a seleção de atributos de personalidade (pelo Agente Social), de forma que o estudante simulado possa adaptar-se de acordo com as necessidades do seu grupo.

2. Permitir a seleção do nível de conhecimento do estudante simulado. $O$ conhecimento pode variar de acordo com a necessidade dos estudantes do grupo.

3. Registrar-se com o agente coordenador de grupos (Comserver, na Figura 2), de forma que possa se comunicar com os demais agentes do ambiente.

4. Solicitar a entrada em um novo grupo de estudo.

5. Editar a Rede Bayesiana do seu grupo ou enviar mensagem de Chat:

1. Verificar a necessidade de Interação:

i. Analisar $\log$ do grupo;

ii. Atualizar os modelos de aluno e de grupo;

iii. Analisar confiança e credibilidade do grupo;

iv. Observar classificação da RB do grupo;

v. Observar personalidade e nível de expertise;

vi. Decidir a ação adequada ao ciclo de interação atual. 


\section{Projeto Interno do Estudante Simulado}

A arquitetura do agente é subdividida em quatro módulos principais: gerenciamento do conhecimento, gerenciamento de ações, comunicação e personalidade.

O módulo de gerenciamento do conhecimento é responsável por atualizar os modelos de aluno e de grupo, além do conhecimento do estudante simulado. Esse módulo foi construído estendendo a abordagem proposta por Vizcaíno (2002), que propõe uma arquitetura genérica para estudantes simulados. Nesse modelo, o estudante simulado tem o mesmo status do estudante real, interagindo com a interface do editor colaborativo. O diagrama do módulo de gerenciamento do conhecimento é apresentado na Figura 4.

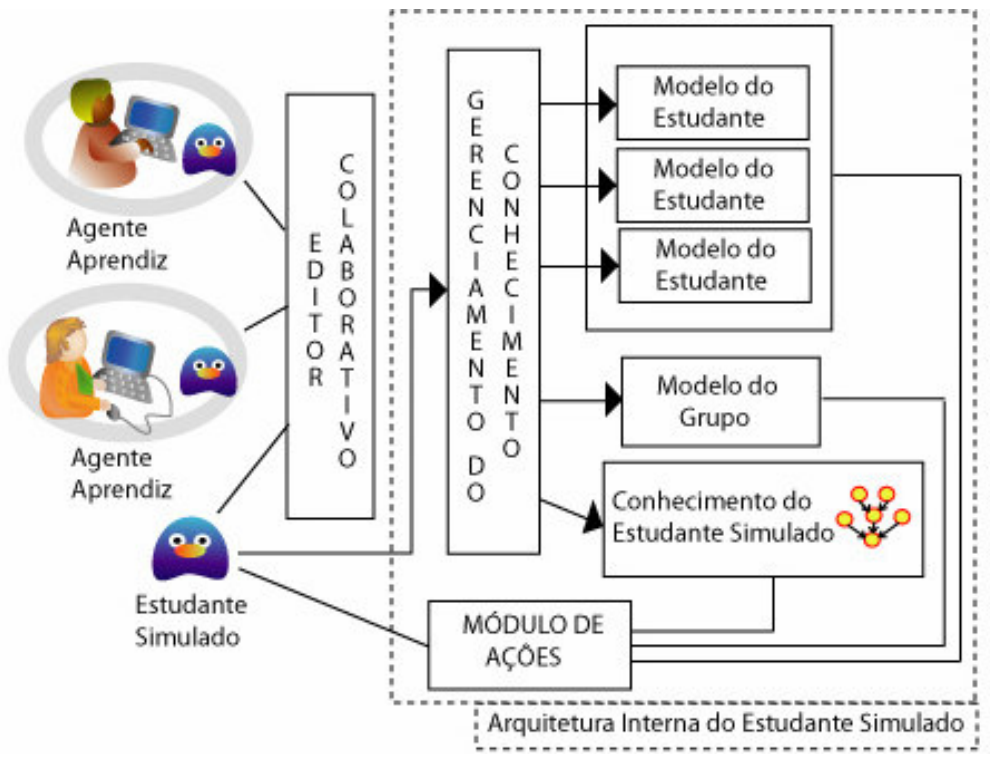

Figura 4 - Módulo de Gerenciamento do Conhecimento do Estudante Simulado.

O módulo de personalidade utiliza a modelagem de estados afetivos proposta por Conati (2003) e adaptada por Boff (2006) para as características aplicáveis ao AMPLIA. Esse modelo baseia-se na teoria cognitiva de emoções OCC (Ortony et al., 1998). A teoria OCC descreve as emoções como resultado do julgamento de como uma situação se ajusta aos objetivos e preferências de uma pessoa. A Figura 5 apresenta a rede do modelo cognitivo do aluno, mostrando traços de personalidade e objetivos.

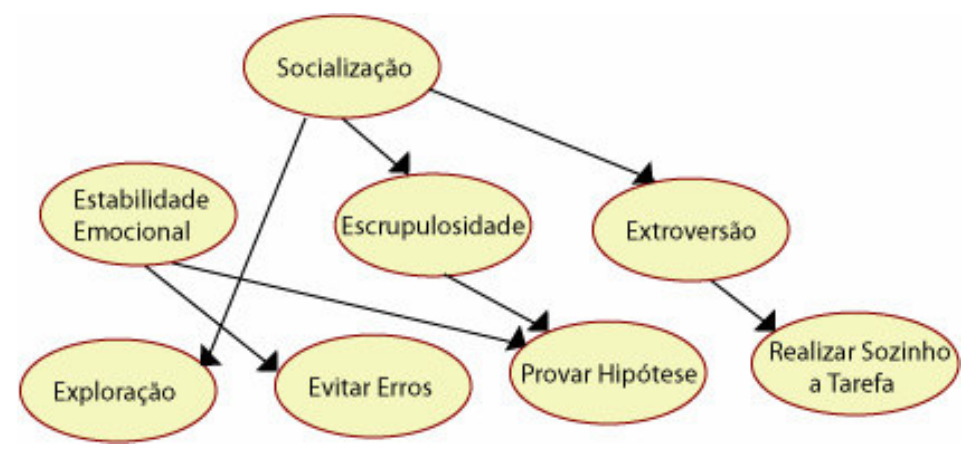

Figura 5 - Rede Bayesiana do modelo do aluno para o estudante simulado: traços de personalidade e objetivos. 
Por meio da modelagem de estados afetivos, o estudante simulado apresenta características afetivas de um estudante real. Isso permite que o estudante simulado interaja de forma mais natural com os estudantes reais. No treinamento de tutores, o comportamento similar ao de um estudante real permite experiências de tutoria mais realísticas.

O módulo de ações recebe as informações armazenadas pelo módulo de gerenciamento do conhecimento e de personalidades e define o plano de ação do estudante simulado. A deliberação é feita por meio da seleção de ações representadas por um diagrama de influências.

O Agente Estudante Simulado utiliza aprendizagem por reforço, atribuindo maior utilidade àquelas ações que têm efeito positivo sobre o grupo. Isso é necessário porque as utilidades iniciais (assim como todos os dados quantitativos das redes probabilísticas) são definidas de forma empírica, devido à impossibilidade de quantificar de forma exata os parâmetros da aprendizagem humana. Além disso, a efetividade das ações está fortemente relacionada a características particulares de um grupo.

O módulo de comunicação define as mensagens a serem trocadas com os demais agentes do AMPLIA. As mensagens seguem o padrão FIPA (2007) e são definidas de acordo com as responsabilidades do Agente Estudante Simulado, exibidas na Tabela 2.

\section{Trabalhos Relacionados}

A maioria das pesquisas em estudantes simulados está na área de avaliação de material educacional. Isso pode ser explicado pela possibilidade de restrição da linguagem utilizada para avaliar os materiais a serem desenvolvidos. Alguns trabalhos que exploram o conceito de avaliação de materiais são o de Virvou e Manos (2003), que apresenta avaliação da autoria de jogos educacionais em uma ferramenta de autoria baseada em bases de conhecimento, e o de Abdullah e Cooley (2002), que aplica a avaliação a um sistema de testes adaptativos. O trabalho de Beck (2002) utiliza um estudante simulado para avaliar um Sistema Tutor Inteligente.

$\mathrm{Na}$ área de aprendizagem colaborativa, podem-se citar os trabalhos de Barceló (2002) e de Vizcaíno e Boulay (2005) que objetivam estimular a aprendizagem em pares, por meio da atuação do estudante simulado. Essa atuação ocorre por meio de diálogos textuais entre o estudante real e o estudante simulado.

Uma aplicação de estudante simulado que não se classifica nos tipos citados é apresentada por Stathacoupoulo et al. (2004). Seu trabalho propõe a utilização de estudantes simulados para gerar dados que permitem treinar e testar um modelo fuzzy baseado em redes neurais.

\section{Conclusões}

Este trabalho propõe a utilização de técnicas de inteligência artificial para a construção de um estudante simulado. Considerando que os estudantes simulados têm melhor atuação na resolução de problemas em que não é necessária a utilização de linguagem natural e que os trabalhos existentes não utilizam essa característica, este trabalho explora a atuação de estudantes simulados em ambientes de aprendizagem baseados em Redes Bayesianas.

O estudante simulado é construído na forma de um agente autônomo que se integra ao ambiente multiagente de aprendizagem AMPLIA. O Agente Estudante 
Simulado utiliza redes bayesianas dinâmicas para representar o conhecimento do agente no domínio de estudo. Sua tomada de decisão utiliza a extensão do formalismo das redes bayesianas dinâmicas, conhecido como redes de decisão dinâmicas. Por meio dessa abordagem constrói-se um agente robusto que atue em um domínio incerto, como é o caso da aprendizagem humana.

Os próximos passos deste trabalho incluem a realização de oficinas para a análise do comportamento do estudante simulado na participação em grupos de trabalho reais. Resultados preliminares obtidos na realização de um workshop com grupos de estudantes de medicina da Fundação Faculdade Federal de Ciências Médicas de Porto Alegre (agosto de 2007) mostraram o grande interesse por parte dos estudantes em uma edição colaborativa dos casos de estudo e na elaboração de soluções que auxiliem os alunos no processo de desenvolvimento do raciocínio diagnóstico.

\section{Agradecimentos}

Este trabalho é suportado pela agência de fomento CNPq.

\section{Referências}

ABDUlLAH, S., COOLEY, R. 2002. Using Simulated Students to Evaluate an Adaptive Testing System. In International Conference on Computers in Education, Massey University, Auckland, New Zealand, page 5, Massey University, Auckland, New Zealand, December 2002. IEEE Computer Society.

BARCELÓ, A.V. 2002. A Simulated Student Model for Improving Collaborative Learning. In: Technologies de l'Information et de la Communication dans les Enseignements d'ingénieurs et dans l'industrie. Villeurbanne : Institut National des Sciences Appliquées de Lyon. 273-281.

BECK, J. E. 2002. Directing Development Effort with Simulated Students. Sixth International Conference on Intelligent Tutoring Systems (ITS'2002), June, 2002.

BOFF, E., SANTOS, E. R., AND VICARI, R. M. 2006. Social Agents to Improve Collaboration on an Educational Portal. In Proceedings of the Sixth IEEE International Conference on Advanced Learning Technologies (July 05 - 07, 2006). ICALT. IEEE Computer Society, Washington, DC, 896-900.

BURLESON, W. 2004. Affective Learning Companions. Doctorial Consortium in Conjunction with the 7th International Conference on Intelligent Tutoring Systems, August 30, 2004, Maceio - Alagoas, Brasil.

CHOU, CHIH-YUEH, CHAN, TAK-WAI, LIN,CHI-JEN. 2002. An Approach of Implementing General Learning Companions for Problem Solving, IEEE Transactions on Knowledge and Data Engineering, v.14 n.6, p.1376-1386, November 2002.

DILLENBOURG P. (1999) What do you mean by collaborative learning?. In P. Dillenbourg (Ed) Collaborative-learning: Cognitive and Computational Approaches. (pp.1-19). Oxford: Elsevier. 
GLUZ, J.C .2005. Formalização da Comunicação de Conhecimentos Probabilísticos em Sistemas Multiagentes: uma abordagem baseada em lógica probabilística. Tese (Doutorado em Ciência da Computação) Instituto de Informática, Universidade Federal do Rio Grande do Sul, Porto Alegre.

HOLMES, JEFFREY THOMAS GRANT. 2003. Learning by Explaining: The Effects of Software Agents as Learning Partners. PhD Dissertation. Vanderbilt University.

MATSUDA, N., COHEN, W. W., \& KOEDINGER, K. R. (2007; to appear). Evaluating a simulated student using real students data for training and testing. In Proceedings of the International Conference on User Modeling.

ORTONY, A. et al. The cognitive structure of emotions. Cambridge Univ. Press, UK. 1998.

PEARL, J. Belief networks revisited, Artificial Intelligence, v.59, n.1-2, p.49-56, 1993.

PETERSON, M. 1997. Skills to Enhance Problem-based Learning. Med Educ Online. 1997; 2,3. URL http://www.med-ed-online/

SEIXAS, L., FLORES, C. D., GLUZ, J. \& VICARI, R. M. (2004). Acompanhamento do processo de construção do conhecimento por meio de um agente probabilístico, In: Anais do 15th Simpósio Brasileiro de Informática na Educação, UFAM, ManausBrazil.

VANLEHN, K., AND OHLSSON, S. 1994. Applications of simulated students: An exploration. Journal of Artificial Intelligence in Education 5(2):135--175.

VICARI, R. M. FLORES, C. D., SEIXAS, L., SILVESTRE, A., LADEIRA, M. \& COELHO, H. (2003). A Multi-Agent Intelligent Environment for Medical Knowledge. In: Journal of Artificial Intelligence in Medicine, Vol.27. Elsevier Science, Amsterdam, 335-366.

VIRVOU, M., AND MANOS, K. 2003. A Simulated Student-Player in Support of the Authoring Process in a Knowledge-Based Authoring Tool for Educational Games. International Conference on Advanced Learning Technologies 2003: 338-339.

VIZCAÍNO, A.,DU BOULAY, B. 2002. Using a Simulated Student to Repair Difficulties in Collaborative Learning. ICCE 2002: 349-353.

WEBB, N. 1989. Peer interaction and learning in small groups. International Journal of Educational Research, 13, 21-40.

WOOLDRIDGE, M. Intelligent Agents, In: Weiss, G. Multiagent Systems, Cambridge, MA, Estados Unidos: The MIT Press, 1999. p.27-72 